\title{
Surface-sensitive spectro-electrochemistry using ultrafast 2D ATR IR spectroscopy
}

\author{
Lotti, Davide ; Hamm, Peter ; Kraack, Jan Philip
}

\begin{abstract}
A new method is presented for the combination of spectro-electrochemistry and femtosecond 2D IR spectroscopy. The key concept is based on ultrathin (similar to $\mathrm{nm}$ ) conductive layers of noble metals and indium-tin oxide (ITO) as working electrodes on a single-reflection attenuated total reflectance (ATR) element in conjunction with ultrafast, multidimensional ATR spectroscopy. The ATR geometry offers prominent benefits in ultrafast spectro-electrochemistry, that is, surface sensitivity for studying electrochemical processes directly at the solvent-electrode interface as well as the application of strongly IR-absorbing solvents such as water due to a very short effective path length of the evanescent wave at the interface. We present a balanced comparison between usable electrode materials regarding their performance in the ultrafast ATR setup. The electrochemical performance is demonstrated by vibrational Stark-shift spectroscopy of carbon monoxide (CO) adsorbed to platinum-coated, ultrathin ITO electrodes. We furthermore measure vibrational relaxation and spectral diffusion of the stretching mode from surfacebound CO dependent on the applied potential to the working electrode and find a negligible impact of the electrode potential on ultrafast $\mathrm{CO}$ dynamics.
\end{abstract}

DOI: https://doi.org/10.1021/acs.jpcc.6b00395

Posted at the Zurich Open Repository and Archive, University of Zurich

ZORA URL: https://doi.org/10.5167/uzh-131992

Journal Article

Accepted Version

Originally published at:

Lotti, Davide; Hamm, Peter; Kraack, Jan Philip (2016). Surface-sensitive spectro-electrochemistry using ultrafast 2D ATR IR spectroscopy. Journal of Physical Chemistry C, 120(5):2883-2892.

DOI: https://doi.org/10.1021/acs.jpcc.6b00395 


\section{Surface-Sensitive Spectro-Electrochemistry}

\section{Using Ultrafast 2D ATR IR Spectroscopy}

Davide Lotti, Peter Hamm, and Jan Philip Kraack*

Department of Chemistry, University of Zürich, Winterthurerstrasse 190, CH-8057

\section{Corresponding Author}

*philip.kraack@chem.uzh.ch

Phone: +41 446354477

Fax: +41446356838

\section{TOC Graphic}

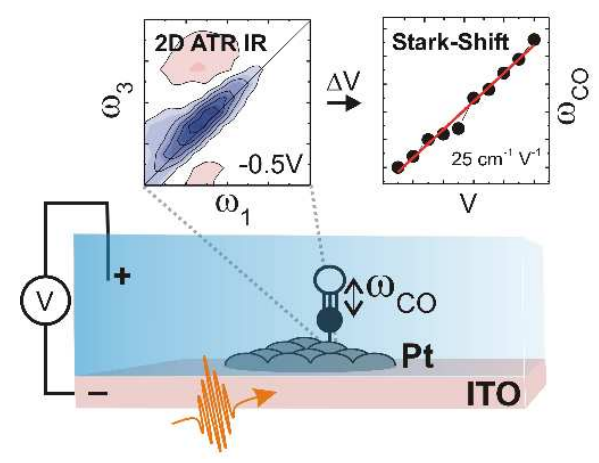

\section{Keywords:}

Spectro-Electrochemistry, Stark-Shift Spectroscopy, Attenuated Total Reflectance Spectroscopy, 2D ATR IR spectroscopy, Carbon monoxide, Nanoparticles, Surface Spectroscopy 


\section{ABSTRACT}

A new method is presented for the combination of spectro-electrochemistry and femtosecond 2D IR spectroscopy. The key-concept is based on ultrathin $(\sim \mathrm{nm})$ conductive layers of Indium-Tin-Oxide (ITO) as working electrodes on a single reflection Attenuated Total Reflectance (ATR) element in conjunction with ultrafast, multi-dimensional ATR spectroscopy. The ATR geometry offers prominent benefits as compared to transmission or external reflection experiments in ultrafast spectroelectrochemistry, i.e. surface-sensitivity for studying electrochemical processes directly at the solventelectrode interface as well as the application of strongly IR-absorbing solvents such as water due to a very short effective path-length of the evanescent wave at the interface. We present $2 \mathrm{D}$ ATR IR spectra of carbon monoxide (CO) adsorbed to platinum-coated, ultrathin ITO electrodes with the electrochemical performance being demonstrated by vibrational Stark-shift spectroscopy of the CO stretching mode. 
The dynamics of molecules at solid-liquid interfaces, which can be electrified on demand, are of significant importance in different fields of chemistry and physics, but are difficult to access experimentally. ${ }^{1-7}$ Particularly ultrafast vibrational spectroscopy offers a high potential regarding identification and characterization of transient species at electrode-electrolyte interfaces. These signals bear information of the structural dynamics of and interaction between molecules and their environment. It is well-known that two-dimensional infrared (2D IR) spectroscopy ${ }^{8-11}$ contains such detailed information. In analogy to 2D NMR spectroscopy, a correlation between two frequency axes allows for the resolution of for instance vibrational coupling, chemical exchange, energy transfer, vibrational relaxation or spectral diffusion of chemical bonds in 2D IR spectroscopy. ${ }^{8-11}$ It is highly desirable to make such information available also from electrode-electrolyte interfaces.

Here, we report a new spectroscopic method for the resolution of ultrafast, multi-dimensional signals from immobilized molecules at electrodes in solution. Our method is based on Attenuated Total Reflectance (ATR) spectro-electrochemistry ${ }^{12-14}$ in combination with the recently developed ultrafast 2D (ATR) IR spectroscopy ${ }^{15-17}$ and yields interface-sensitive, multi-dimensional signals with sub-picosecond temporal resolution. We resolve 2D ATR IR signals from carbon monoxide $(\mathrm{CO})$ adsorbed from aqueous solution on Platinum $(\mathrm{Pt})$-coated ultrathin Indium-Tin-Oxide (ITO) electrodes. Variation of the electrochemical potential applied to the ITO electrode is demonstrated to shift the vibrational frequency of the immobilized $\mathrm{CO}$ with a rate of $\sim 25 \mathrm{~cm}^{-1} \mathrm{~V}^{-1}$ on the basis of vibrational Stark-shift spectroscopy $^{18,19}$.

Figure 1 shows a schematic drawing of the home-built electrochemical ATR cell for ultrafast experiments. The assembly consists of a right-angle, single reflection $\mathrm{CaF}_{2}$ prism (top surface $10 \times 14 \mathrm{~mm}$, Thorlabs), the reflecting plane of which is sputter-coated with an ultrathin layer of ITO that is used as the working electrode (vide infra). The ITO layer is incorporated in an 
electrochemical circuit containing a potentiostat (IviumStat. Inc.) via a thin adhesive aluminum foil (Electron Microscopy Science Inc.) sandwiched between the cell and the ITO surface. Additionally, the potentiostat is connected to counter- and reference electrodes, which are assembled inside a home-built poly-ether-ether-keton (PEEK) sample flow-cell. The counter electrode consists of a Pt-coated Ti-wire (E-DAQ Inc.) and is centered in the chamber of the flow-cell. The reference electrode $(\mathrm{Ag} / \mathrm{AgCl}$, Biosense Inc.) is located at the front side of the PEEK cell.

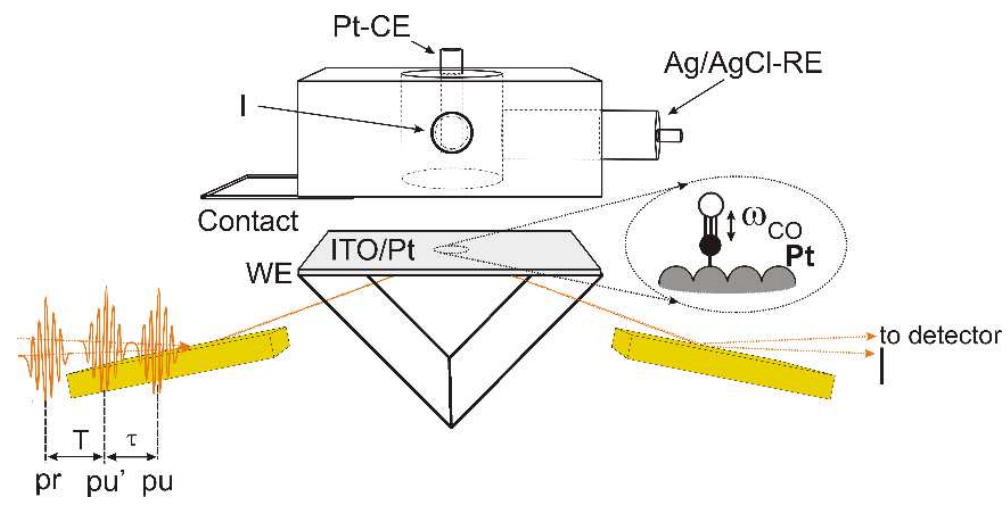

Figure 1. Sketch of the electrochemical ATR cell used in the ultrafast 2D ATR IR measurements. Carbon monoxide $(\mathrm{CO})$ is adsorbed on ultrathin Platinum (Pt)-coated IndiumTin-Oxide (ITO) electrodes. Two collinear pump pulses are focused on the reflecting plane of a single reflection ATR cell, excite the adsorbed molecules and a delayed, weak probe pulse interrogates the dynamics. The electrochemical cell consists of a working electrode (WE), a counter electrode $(\mathrm{CE})$ and a reference electrode $(\mathrm{RE})$. The electrochemical circuit involving the potentiostat is not shown for clarity. Prism and cell elements are not drawn to scale for clarity.

The sample cell assembly is mounted on top of the reflecting plane of the ITO/Pt-coated ATR prism using an O-ring (Teflon) to allow solvent flow above the working electrode. The O-ring has an inner diameter of $7 \mathrm{~mm}$, which determines the area of the electrochemically active 
surface. The sample chamber of the flow-cell is centered above the reflecting plane of the ATR prism and has an inner diameter of about $5 \mathrm{~mm}$ and a volume of about $0.5 \mathrm{~cm}^{3}$. In- and outlets of the sample cell (I/O) are located on opposite sides of the flow-cell and are connected to Teflon tubes (outlet not shown in Fig. 1).

The ultrafast ATR arrangement is the same as employed previously for the 2D ATR IR spectroscopy of, e.g. organic monolayers (MLs) on thin metal layers. ${ }^{15,16} \mathrm{CaF}_{2}$ is used as an ATR material due to its broad transparency in the mid-IR region (down to $\sim 1100 \mathrm{~cm}^{-1}$ ). In addition, more widely applied ATR crystals such as Germanium, Silicon or Zinc-Selenide possess significantly lower bandgaps of electronic excitation, which make these materials susceptible to multi-photon absorption of the intense IR pulses. The comparatively low refractive index $(\mathrm{n}=1.41)$ of $\mathrm{CaF}_{2}$ as compared to water $(\mathrm{n}=1.31)$ makes it necessary to use a very large value for the angle of incidence $\left(\sim 85^{\circ}\right)$ in order to satisfy total internal reflection conditions, as described previously ${ }^{17}$.

Conductive layers as working electrodes on the ATR prism are obtained by use of ITO as an electrode material instead of thin metal layers such as Gold or Platinum, which are generally employed in stationary ATR IR spectro-electrochemistry. ${ }^{12-14}$ This is due to the better mechanical stability of ITO as opposed to noble metals which often require a second metal interlayer such as Chromium or Titanium for adhesion purposes. ${ }^{20-22}$ In addition, ITO exhibits a higher transparency of mid-IR $\operatorname{light}^{23}$ as compared to similarly thin metal layers. This is beneficial in ultrafast time-resolved spectroscopy since the lower absorption of ITO guarantees highest intensity of the IR light at the electrode-solvent interface, resulting in maximum signal intensity from the immobilized sample. The stronger stationary absorption of metal layers in the mid-IR also results in large background signals which obscure the sought signals from the adsorbates. Testing of different thicknesses in the range of $1-20 \mathrm{~nm}$ ITO in 
the ultrafast 2D ATR IR experiment, we found that an average thickness of $2 \mathrm{~nm}$ yields the optimal balance between electrical conductivity and signal intensity.

The ultrathin ITO layers are sputter-coated on the reflecting plane of the $\mathrm{CaF}_{2}$ ATR prism using $\mathrm{Ar}^{+}$-ion sputtering (Bal-Tec SCD500, ZMB, Zürich) at a working distance of $50 \mathrm{~mm}$, a base-pressure of $8 \times 10^{-5}$ mbar and a working pressure of $8 \times 10^{-3}$ mbar. For the deposition of ITO, a sputtering current of $60 \mathrm{~mA}$ was used which results in a deposition rate of about $0.15 \mathrm{~nm} \mathrm{~s}^{-1}$. The average thickness was in-situ monitored using a quartz microbalance. The sputtering process results in a structured surface of ITO nanoparticles (NPs) as determined by scanning electron microscopy analysis (SEM, Zeiss Supra 50 VP, Fig. 2). The SEM analysis reveals that the NPs (light grey) form a heterogeneous surface of islands with shortest dimensions of $\sim 5 \mathrm{~nm}$ and longest extensions of $50-100 \mathrm{~nm}$. These islands are partly interconnected with distances between the islands, which is estimated from the SEM images to be at maximum $5 \mathrm{~nm}$ (dark grey). In this form, the sputtered ITO layers have a typical resistance of about $1 \mathrm{k} \Omega$ measured with two tips of a tester separated by about $1 \mathrm{~cm}$, which is sufficiently low for a quick equilibration of the electrochemical potential on the surface.

Ultrathin (average thickness of $0.3 \mathrm{~nm}$ ) Pt NPs are sputter-coated on top of the ITO electrodes using a sputtering current of $6 \mathrm{~mA}$, which resulted in a deposition rate of about $0.02 \mathrm{~nm} \mathrm{~s}^{-1}$. Otherwise, the deposition parameters were the same for ITO (vide supra). Carbon monoxide (CO) saturated solutions were prepared by bubbling CO (4.8 grade, Pangas) for 15 minutes through a double distilled water solution containing $0.1 \mathrm{M} \mathrm{NaClO}_{4}$ (puriss. p.a., Sigma Aldrich) as electrolyte. The prepared CO solutions were flown across the electrode for a few minutes until adsorption was complete. ${ }^{17}$ 


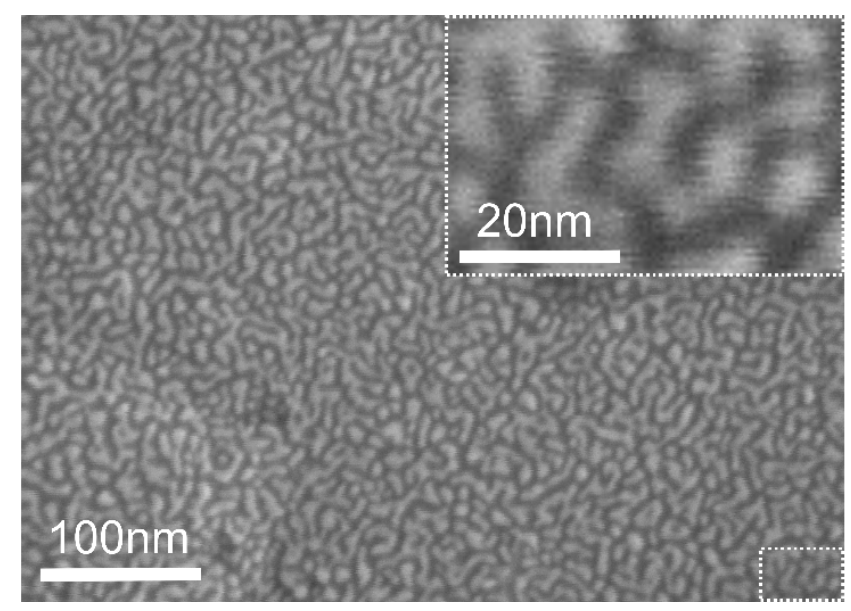

Figure 2. Scanning Electron Microscopy (SEM) image of a sputter-coated, ultrathin ITO film (average thickness $2 \mathrm{~nm}$ ) on the surface of a $\mathrm{CaF}_{2}$ substrate used as electrodes in the spectroelectrochemistry experiments. The film consists of ITO nanoparticles (light grey) which are partly interconnected near the percolation threshold. The upper right inset shows a magnification of the lower right section by a factor of five.

For 2D ATR IR measurements, the experimental setup is the same as described previously. ${ }^{15-}$ 17 In brief, $\sim 90 \mathrm{fs}$ mid-IR pulses $\left(\sim 250 \mathrm{~cm}^{-1}\right.$ bandwidth) centered around $2000 \mathrm{~cm}^{-1}$ are generated from an OPA, ${ }^{24}$ which is pumped by a $5 \mathrm{kHz}$ regenerative amplifier at $800 \mathrm{~nm}$. The output energy is about $1.5 \mu \mathrm{J}$ per pulse. The major portion is directed to a Mach-Zehnder interferometer to obtain coherent pump pulse pairs for 2D IR spectroscopy. About $5 \%$ of the OPA output is split off with a $\mathrm{BaF}_{2}$ wedge and used as probe and reference beams for balanced signal detection on a $2 \times 32$ pixel MCT array detector mounted to a spectrograph. Pump-, probe- and reference beams are focused on the backside of the reflecting plane of the ATR crystal by use of off-axis parabolic mirrors. 2D spectra are generated by scanning the coherence time ( $\tau$, Fig. 1) between the two pump pulses with a fixed population time (T, Fig. 1) between the second pump pulse and the probe pulse. A succeeding Fourier-transformation of the oscillating signal part ${ }^{25}$ yields the 2D IR spectrum. 
Linearly bound $\mathrm{CO}$ on Pt-NPs exhibits a strong IR absorption band, which sensitively responds to electrochemical potentials of the electrode. ${ }^{26,27}$ By changing the potential, the $\mathrm{CO}$ stretch frequency can be varied in a potential range of -0.5 to about $+0.4 \mathrm{~V}$. In this potential range $\mathrm{CO}$ is stable on small $\mathrm{Pt}-\mathrm{NPs}$, but complete $\mathrm{CO}$ desorption is observed for potentials larger than $+0.4 \mathrm{~V} \cdot{ }^{26,27}$ Fig. 3 shows 2D ATR IR spectra of CO on Pt for a population delay of 2 ps and for three potentials, i.e. $-0.5 \mathrm{~V}$ (a), $0.0 \mathrm{~V}(\mathrm{~b})$, and $+0.3 \mathrm{~V}$ (c). These signals have been obtained by subtraction of two separately measured spectra of the electrolyte solution on the ITO electrodes with an without $\mathrm{CO} .{ }^{17}$ Note that the signal intensity of the $+0.3 \mathrm{~V} 2 \mathrm{D}$ ATR IR spectrum has been multiplied by a factor of 2.5 in order to compensate for the already partial desorption of $\mathrm{CO}$ at that potential. ${ }^{26-28}$ Ground-state bleach signals (blue) are predominately observed together with induced excited state absorption signals (red). The ground-state bleach signals are strongly elongated along the diagonal, indicating a large degree of inhomogeneity in the vibrational lineshape of the immobilized $\mathrm{CO}$, as discussed previously ${ }^{17}$. Note that induced absorption signals appear both red and blue-shifted with respect to the ground-state bleach signal. These contributions stem from well-known dispersive lineshapes of ATR signals ${ }^{29,30}$ and their influence on 2D ATR IR spectra will be discussed in detail elsewhere. They are thus artificial signals which obscure the expected ${ }^{17}$ exclusively red-shifted excited-state absorption signals. As the potential is varied from negative $(-0.5 \mathrm{~V},(\mathrm{a}))$ to positive $(+0.3 \mathrm{~V},(\mathrm{c}))$ values, the central frequency of the $\mathrm{CO}$ band changes between $2050-2070 \mathrm{~cm}^{-1}$. Recording the $\mathrm{CO}$ band maxima projected on the probe axis at a series of different potentials (Fig. 3 (d)) reveals that the central frequency (open circles) is shifted upon potential variation in a linear manner. A linear fit to the data gives a slope of $\sim 25 \mathrm{~cm}^{-1} \mathrm{~V}^{-1}$ which is in good agreement with previous results $\left(25-30 \mathrm{~cm}^{-1} \mathrm{~V}^{-1}\right)^{26-}$ 28. The origin of this spectral shift in known as a vibrational Stark-effect ${ }^{18,26,31,32}$ which is based on alterations of the vibrational properties and chemical binding properties of adsorbates in electric fields near electrodes. 

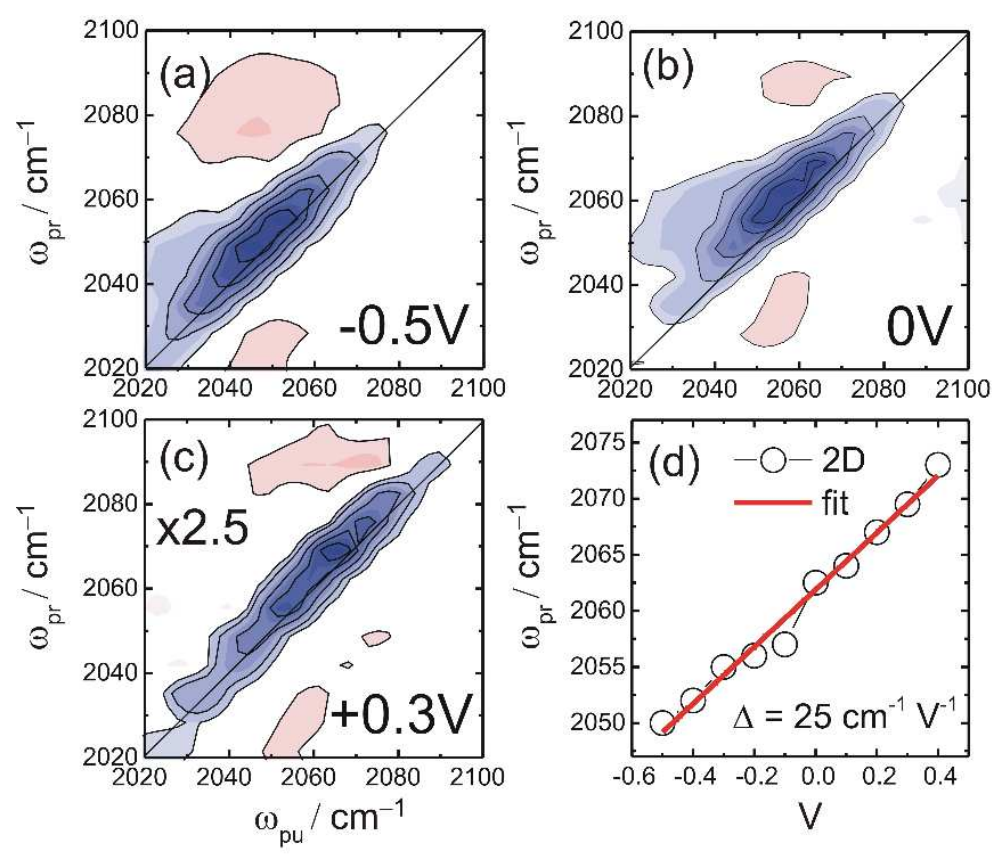

Figure 3. 2D ATR IR signals of CO absorbed on ultrathin ITO/Pt electrodes for different potentials and a population time $\mathrm{T}=2 \mathrm{ps}$. (a) $-0.5 \mathrm{~V}$, (b) $0 \mathrm{~V}$, (c) $+0.3 \mathrm{~V}$. Note that the $2 \mathrm{D}$ spectrum in (c) is multiplied by a factor of 2.5 due to partial desorption of $\mathrm{CO}$ at that potential. (d) Spectral positions of the ground-state bleach (blue) CO band maxima projected on the probe axis against the applied potential. Open circles are experimental data, the solid red line is a linear fit with a slope $\Delta=25 \mathrm{~cm}^{-1} \mathrm{~V}^{-1}$.

The 2D ATR IR measurements presented above demonstrate the applicability of our electrochemical cell in a vibrational Stark-shift experiment of surface-bound molecules. Making available ultrafast IR signals of samples at electrode interfaces is valuable in many ways. That is, spectro-electrochemistry in conjunction with time-resolved spectroscopy is an intense field of research for instance concerning redox-related bio-chemical processes ${ }^{14}$, electro-catalytic reactions $s^{33}$ or solar cells ${ }^{4}$. In these disciplines, very often questions emerge which relate to vibrational, orientational and energy-transfer dynamics of molecules in direct 
contact with, or in the vicinity of electrode-solvent interfaces. Ultrafast IR spectroscopy allows one to resolve these dynamics with sub-picosecond temporal resolution. ${ }^{8-11}$ Particularly 2D IR spectroscopy offers the possibility to directly investigate also intermolecular dynamics of molecules such as vibrational coupling ${ }^{34}$ or chemical exchange ${ }^{35}$, details of which are largely unexplored to date for interfacial systems, and in particular near electrodes. In this context, we note that 2D ATR IR spectroscopy has already been demonstrated to be sensitive enough to detect organic monolayers equipped with only lowabsorbing $\left(\varepsilon<200 \mathrm{M}^{-1} \mathrm{~cm}^{-1}\right)$ local vibrational probes even at sub-monolayer coverages. ${ }^{15,16}$

Time-resolved spectroscopy at charged interfaces gained considerable attention in recent years. .,7,28,36-39 Most studies concerning vibrational spectroscopy employ ultrafast SumFrequency-Generation (SFG) spectroscopy which is based on a combination of mid-IR and visible beams..$^{3,7,28,36-39}$ However, recent reports also successfully demonstrated signals obtained exclusively in the mid-IR spectral region. ${ }^{40,41}$ Given the need of a solid state interface working as an electrode, experimental arrangements in transmission have not yet been reported for ultrafast spectroscopy to the best of our knowledge. Alternatively, one may use designs with either an external ${ }^{40,41}$ or an internal (i.e., ATR) reflection geometry. ${ }^{5,12-14}$ When working with exclusively mid-IR light in external reflection conditions, the excitation light travels through the (generally) aqueous electrolyte solution twice which demands liquid layers of only a few microns in thickness, due to the broad and strong IR absorbance of water. $^{40,41}$ This is less of an issue in external reflection SFG spectroscopy, since the signal light is up-converted to the visible spectral range at the interface. In ATR spectroscopy, in contrast, challenges concerning sample thicknesses are circumvented due to the very low penetration depth of the evanescent wave at the interface. The highest intensity of the evanescent field is present directly at the solid-liquid interface, giving rise to the well-known surface-sensitivity of ATR. As a rule of thumb, the evanescent fields produced at the interface 
exhibit a penetration depth of generally about one wavelength of the applied light (here about $5 \mu \mathrm{m}) .{ }^{42}$ Moreover, the penetration-depth can easily be tuned to both smaller and larger values by varying the angle of incidence of the IR light. ${ }^{42}$ As a result of the properties of the evanescent wave, ATR is thus still applicable for the investigation of electrochemically active samples in bulk solution since the method depends on the third-order nonlinear susceptibility of the sample. Moreover, ATR is expected to be able to resolve dynamics from samples not only from the interface but also from, e.g. molecules within the Helmholtz double layer ${ }^{43}$. In this regard, a major future extension of the current status of our setup will involve the transfer of redox-active samples from bulk solution to organic monolayers ${ }^{15,16}$ in order to allow investigations of electro-catalytic reactions ${ }^{44}$ or potential dependent orientational dynamics ${ }^{45}$.

In conclusion, we have presented a new spectroscopic method for measuring multidimensional, time-resolved IR spectra from immobilized molecules at electrode-electrolyte interfaces. Using a combination of electrochemical ATR spectroscopy with ultrathin conductive layers and the recently developed 2D ATR IR spectroscopy, multi-dimensional vibrational Stark-shift spectra of carbon monoxide (CO) adsorbed to Platinum nanoparticles on ultrathin, nanostructured Indium-Tin-Oxide electrodes have been acquired. In a potential window of $-0.5 \mathrm{~V}-+0.4 \mathrm{~V}$ the $\mathrm{CO}$ band was shifted at a rate of $\sim 25 \mathrm{~cm}^{-1} \mathrm{~V}^{-1}$ and electrochemical desorption was observed for more positive potentials. We expect that ultrafast 2D ATR IR spectro-electrochemistry will find broad application in various research fields which involve purposely charged interfaces as well as redox- and electron-transfer dynamics across solid-liquid interfaces. 


\section{Acknowledgements}

The work has been supported by the Swiss National Science Foundation (grant CRSII2_136205/1) and by the University Research Priority Program (URPP) for solar light to chemical energy conversion (LightChEC). Experimental support by the Center of Microscopy and Imaging at the University of Zürich (ZMB) with regard to the preparation and characterization of ITO layers, as well as by Henrik Braband (University of Zürich) with regard to preparation of $\mathrm{CO}$ solutions, is gratefully acknowledged.

\section{References}

(1) Rodriguez, P.; Kwon, Y.; Koper, M. T. M. The Promoting Effect of Adsorbed Carbon Monoxide on the Oxidation of Alcohols on a Gold Catalyst. Nat. Chem. 2012, 4, 177182.

(2) Bandarenka, A. S.; Koper, M. T. M. Structural and Electronic Effects in Heterogeneous Electrocatalysis: Toward a Rational Design of Electrocatalysts. J. Catal. 2013, 308, $11-24$.

(3) Liu, W.-T.; Shen, Y. R. In Situ Sum-Frequency Vibrational Spectroscopy of Electrochemical Interfaces with Surface Plasmon Resonance. Proc. Natl. Acad. Sci. 2014, 1-5.

(4) Grätzel, M. Photoelectrochemical Cells. Nature 2001, 414, 338-344.

(5) Ashley, K.; Pons, S. Infrared Spectroelectrochemistry. Chem. Rev. 1988, 88, 673-695.

(6) Alibabaei, L.; Brennaman, M. K.; Norris, M. R.; Kalanyan, B.; Song, W.; Losego, M. D.; Concepcion, J. J.; Binstead, R. A.; Parsons, G. N.; Meyer, T. J. Solar Water Splitting in a Molecular Photoelectrochemical Cell. Proc. Nat. Acad. Sci. USA 2013, 110, 20008-20013.

(7) Xiong, W.; Laaser, J. E.; Mehlenbacher, R. D.; Zanni, M. T. Adding a Dimension to the Infrared Spectra of Interfaces Using Heterodyne Detected 2D Sum-Frequency Generation (HD 2D SFG) Spectroscopy. Proc. Natl. Acad. Sci. U. S. A. 2011, 108, 20902-20907. 
(8) Hamm, P.; Zanni, M. Concepts and Methods of 2D Infrared Spectroscopy; Cambridge University Press, 2011.

(9) Fayer, M. D. Dynamics of Water Interacting with Interfaces, Molecules, and Ions. Acc. Chem. Res. 2012, 45, 3-14.

(10) van Wilderen, L. J. G. W.; Bredenbeck, J. Von Ultraschnellen Strukturbestimmungen Bis Zum Steuern von Reaktionen: Mehrdimensionale Gemischte IR/nicht-IRSchwingungsspektroskopie. Angew. Chemie 2015, 127, 2-20.

(11) Arnolds, H.; Bonn, M. Ultrafast Surface Vibrational Dynamics. Surf. Sci. Rep. 2010, $65,45-66$.

(12) Osawa, M. Dynamic Processes in Electrochemical Reactions Studied by SurfaceEnhanced Infrared Absorption Spectroscopy (SEIRAS). Bull. Chem. Soc. Jpn. 1997, $70,2861-2880$.

(13) Delgado, J. M.; Orts, J. M.; Pérez, J. M.; Rodes, A. Sputtered Thin-Film Gold Electrodes for in Situ ATR-SEIRAS and SERS Studies. J. Electroanal. Chem. 2008, $617,130-140$.

(14) Jiang, X.; Zaitseva, E.; Schmidt, M.; Siebert, F.; Engelhard, M.; Schlesinger, R.; Ataka, K.; Vogel, R.; Heberle, J. Resolving Voltage-Dependent Structural Changes of a Membrane Photoreceptor by Surface-Enhanced IR Difference Spectroscopy. Proc. Natl. Acad. Sci. U. S. A. 2008, 105, 12113-12117.

(15) Kraack, J. P.; Lotti, D.; Hamm, P. 2D Attenuated Total Reflectance Infrared Spectroscopy Reveals Ultrafast Vibrational Dynamics of Organic Monolayers at MetalLiquid Interfaces. J. Chem. Phys. 2015, 142, 212413.

(16) Kraack, J. P.; Lotti, D.; Hamm, P. Surface-Enhanced, Multi-Dimensional Attenuated Total Reflectance Spectroscopy. In Proc. of SPIE, Physical Chemistry of Interfaces and Nanomaterials XIV; Sophia C. Hayes; Eric R. Bittner, Eds.; 2015; Vol. 9549, p. 95490S.

(17) Kraack, J. P.; Lotti, D.; Hamm, P. Ultrafast, Multidimensional Attenuated Total Reflectance Spectroscopy of Adsorbates at Metal Surfaces. J. Phys. Chem. Lett. 2014, $5,2325-2329$.

(18) Lambert, D. K. Vibrational Stark Effect of Adsorbates at Electrochemical Interfaces. Electrochim. Acta 1996, 41, 623-630. 
(19) Schkolnik, G.; Salewski, J.; Millo, D.; Zebger, I.; Franzen, S.; Hildebrandt, P. Vibrational Stark Effect of the Electric-Field Reporter 4-Mercaptobenzonitrile as a Tool for Investigating Electrostatics at Electrode/SAM/Solution Interfaces. Int. J. Mol. Sci. 2012, 13, 7466-7482.

(20) Matranga, C.; Guyot-Sionnest, P. Vibrational Relaxation of Cyanide at the Metal/electrolyte Interface. J. Chem. Phys. 2000, 112, 7615.

(21) Love, J. C.; Estroff, L. A.; Kriebel, J. K.; Nuzzo, R. G.; Whitesides, G. M. SelfAssembled Monolayers of Thiolates on Metals as a Form of Nanotechnology. Chem. Rev. 2005, 105, 1103-1169.

(22) Zhang, Z.; Imae, T. Study of Surface-Enhanced Infrared Spectroscopy. J. Colloid Interface Sci. 2001, 233, 99-106.

(23) Granqvist, C. G.; Hultåker, A. Transparent and Conducting ITO Films: New Developments and Applications. Thin Solid Films 2002, 411, 1-5.

(24) Hamm, P.; Kaindl, R. A.; Stenger, J. Noise Suppression in Femtosecond Mid-Infrared Light Sources. Opt. Lett. 2000, 25, 1798-1800.

(25) Helbing, J.; Hamm, P. Compact Implementation of Fourier Transform TwoDimensional IR Spectroscopy without Phase Ambiguity. J. Opt. Soc. Am. B 2010, 28, 171.

(26) Tian, L.; Li, J.-T.; Ye, J.-Y.; Zhen, C.-H.; Sun, S.-G. In Situ FTIR Studies of Coadsorption of $\mathrm{CN}-$ and $\mathrm{CO}$ on $\mathrm{Pt}(110)$ Electrode Surface. J. Electroanal. Chem. 2011, 662, 137-142.

(27) Samjeské, G.; Komatsu, K.; Osawa, M. Dynamics of CO Oxidation on a Polycrystalline Platinum Electrode: A Time-Resolved Infrared Study. J. Phys. Chem. C 2009, 113, 10222-10228.

(28) Lu, G. Q.; Lagutchev, A.; Dlott, D. D.; Wieckowski, A. Quantitative Vibrational SumFrequency Generation Spectroscopy of Thin Layer Electrochemistry: CO on a $\mathrm{Pt}$ Electrode. Surf. Sci. 2005, 585, 3-16.

(29) Bürgi, T. ATR-IR Spectroscopy at the Metal-liquid Interface: Influence of Film Properties on Anomalous Band-Shape. Physical Chemistry Chemical Physics, 2001, 3, 2124-2130.

(30) Miljković, M.; Bird, B.; Diem, M. Line Shape Distortion Effects in Infrared Spectroscopy. Analyst 2012, 137, 3954-3964. 
(31) Chang, S.; Weaver, M. J. I N Situ Infrared Spectroscopy of CO Adsorbed at Ordered Pt( 100)-Aqueous Interfaces: Double-Layer Effects upon the Adsorbate Binding Geometry. J. Phys. Chem. 1990, 94, 5095-5102.

(32) Lambert, D. K. Vibrational Stark Effect of CO on $\mathrm{Ni}(100)$, and $\mathrm{CO}$ in the Aqueous Double Layer: Experiment, Theory, and Models. J. Chem. Phys. 1988, 89, 3847.

(33) Russell, A. E. Electrocatalysis: Theory and Experiment at the Interface. Phys. Chem. Chem. Phys. 2008, 10, 3607-3608.

(34) Khalil, M.; Demirdöven, N.; Tokmakoff, A. Coherent 2D IR Spectroscopy: Molecular Structure and Dynamics in Solution. J. Phys. Chem. A 2003, 107, 5258-5279.

(35) Zheng, J.; Kwak, K.; Asbury, J.; Chen, X.; Piletic, I. R.; Fayer, M. D. Ultrafast Dynamics of Solute-Solvent Complexation Observed at Thermal Equilibrium in Real Time. Science 2005, 309, 1338-1343.

(36) Lagutchev, A.; Lu, G. Q.; Takeshita, T.; Dlott, D. D.; Wieckowski, A. Vibrational Sum Frequency Generation Studies of the $(2 \times 2) \rightarrow(\sqrt{19} \times \sqrt{19})$ Phase Transition of CO on Pt(111) Electrodes. J. Chem. Phys. 2006, 125, 154705.

(37) Lis, D.; Backus, E. H. G.; Hunger, J.; Parekh, S. H.; Bonn, M. Liquid Flow along a Solid Surface Reversibly Alters Interfacial Chemistry. Science 2014, 344, 1138-1142.

(38) Eftekhari-Bafrooei, A.; Borguet, E. Effect of Surface Charge on the Vibrational Dynamics of Interfacial Water. J. Am. Chem. Soc. 2009, 131, 12034-12035.

(39) Singh, P. C.; Nihonyanagi, S.; Yamaguchi, S.; Tahara, T. Ultrafast Vibrational Dynamics of Water at a Charged Interface Revealed by Two-Dimensional HeterodyneDetected Vibrational Sum Frequency Generation. J. Chem. Phys. 2012, 137, 094706.

(40) El Khoury, Y.; Wilderen, L. J. G. W. Van; Bredenbeck, J. Ultrafast 2D-IR Spectroelectrochemistry of Flavin Mononucleotide. J. Chem. Phys. 2015, 212416.

(41) El Khoury, Y.; van Wilderen, L. J. G. W.; Vogt, T.; Winter, E.; Bredenbeck, J. A Spectroelectrochemical Cell for Ultrafast Two-Dimensional Infrared Spectroscopy. Rev. Sci. Instrum. 2015, 86, 083102.

(42) Zaera, F. Probing Liquid/solid Interfaces at the Molecular Level. Chem. Rev. 2012, 112, 2920-2986. 
(43) Sugino, O.; Hamada, I.; Otani, M.; Morikawa, Y.; Ikeshoji, T.; Okamoto, Y. FirstPrinciples Molecular Dynamics Simulation of Biased Electrode/solution Interface. Surf. Sci. 2007, 601, 5237-5240.

(44) Chen, Z.; Concepcion, J. J.; Jurss, J. W.; Meyer, T. J. Single-Site, Catalytic Water Oxidation on Oxide Surfaces. J. Am. Chem. Soc. 2009, 131, 15580-15581.

(45) Chidsey, C. E. D.; Bertozzi, C. R.; Putvinski, T. M.; Mujsce, A. M. Coadsorption of Ferrocene-Terminated and Unsubstituted Alkanethiols on Gold : Electroactive SelfAssembled Monolayers. J. Am. Chem. Soc. 1990, 112, 4301-4306. 